\title{
Localization of strongly correlated electrons as Jahn-Teller polarons in manganites
}

\author{
Y.-F. Yang and K. Held \\ Max-Planck-Institut für Festkörperforschung, 70569 Stuttgart, Germany
}

(Dated: March 21, 2006)

\begin{abstract}
A realistic modeling of manganites should include the Coulomb repulsion between $e_{g}$ electrons, the Hund's rule coupling to $t_{2 g}$ spins, and Jahn-Teller phonons. Solving such a model by dynamical mean field theory, we report large magnetoresistances and spectra in good agreement with experiments. The physics of the unusual, insulating-like paramagnetic phase is determined by correlated electrons which are - due to strong correlations - easily trapped as Jahn-Teller polarons.

PACS numbers: 71.30.+h, 71.27.+a, 75.47.Gk
\end{abstract}

Since large magnetoresistances are of substantial technological importance, the colossal magnetoresistance (CMR) 1] in manganites $\left(\mathrm{T}_{1-x} \mathrm{D}_{x} \mathrm{MnO}_{3} ; \mathrm{T}\right.$ : trivalent ion, e.g., La; D: divalent ion, e.g., Ca) has attracted considerable attention, both experimentally and theoretically 2]. Like the mechanism for high-temperature superconductivity in the cuprates, the CMR still lacks a thorough theoretical understanding; and like superconducting transition temperatures, the temperatures for the CMR are much lower than technologically desirable.

The key to the understanding of CMR lies in the unusual properties of the paramagnetic (PM) phase which - in a wide range of dopings $x$ - shows an unusual insulating-like behavior. At lower temperatures $T$ and/or within magnetic fields, the double exchange mechanism [3] stabilizes a ferromagnetic (FM) metallic phase. If the insulating-like PM phase was understood, a CMR could simply be described as the PM-insulator-to-FMmetal transition. Different proposals aim at explaining the CMR and the PM insulating-like phase, in particular, the localization of charge carriers through lattice 4] or orbital 5] polarons, an Anderson localization arising from disordered $t_{2 g}$-spins 6 ], a phase separation into nano-domains showing percolation effects [7, 8], and an effective two band model [9]. However, hitherto no quantitative, microscopic calculation satisfactorily explains the known physical properties of the PM insulating-like phase, which is not only characterized by an increase of the resistivity $\rho$ with decreasing $T$, but also has unusual dynamic properties. The latter reflect in a spectral function $A(\omega)$ with a very low spectral weight at the Fermi level $E_{F}$ irrespectively of $x$, as indicated by photoemission and X-ray absorption experiments 10,11]. Similarly the optical conductivity $\sigma(\omega)$ shows a very low spectral weight up to an energy scale of $\sim 1 \mathrm{eV}$ [12]. Also the FM is an atypical (bad) metal.

The theoretical understanding is complicated since several ingredients seem to be necessary to describe manganites: the exchange interaction between more localized $t_{2 g}$ spins and the itinerant $e_{g}$ electrons [3], the Jahn-Teller phonons and their coupling to the electrons [4], as well as the electronic correlations due to the local Coulomb interaction [13, 14]. The necessity of all three of these interactions was also revealed by realistic LDA+DMFT (local density approximation + dynamical mean field theory 15]) calculations for the parent compound $\mathrm{LaMnO}_{3}$, showing the metal-insulator transition in $\mathrm{LaMnO}_{3}$ under pressure [16] to be triggered by the (Jahn-Teller) crystal field splitting which is strongly enhanced by the local Coulomb interaction [17].

In this Letter, we model doped $\mathrm{LaMnO}_{3}$ by including a realistic tight-binding band structure for cubic $\mathrm{LaMnO}_{3}$, the Coulomb interaction between the $e_{g}$ electrons, JahnTeller phonons, and the Hund's exchange coupling to localized $t_{2 g}$ spins. We solve this model by dynamical mean field theory (DMFT) [18], employing the (numerically) exact quantum Monte Carlo (QMC) 19] method as an impurity solver. We find that tendencies of the Coulomb repulsion and the Jahn-Teller distortion to localize electrons mutually support each other, trapping $e_{g}$ electrons as local polarons in the PM phase. Hence the PM phase is insulating-like with very low optical spectral weight below $1 \mathrm{eV}$ and with a resistivity which is by a factor of $\sim 8$ larger than that of the FM phase (magnetoresistance). Jahn-Teller polarons without correlation effects have been studied before, e.g. in [4, 20, 21], but suffered from shortcomings such as big magnetoresistances only for undoped $\mathrm{LaMnO}_{3}$ (which is always insulating in experiments) and discrepancies with experimental spectra.

Model for manganites. Starting point of our investigation is the following, realistic model for manganites

$$
\begin{aligned}
\hat{H}= & -\sum_{l, m=1}^{2} \sum_{\langle i j\rangle \sigma} t_{l m}^{i j} \hat{c}_{i l \sigma}^{\dagger} \hat{c}_{j m \sigma}-2 \mathcal{J} \sum_{m i} \hat{\mathbf{s}}_{i m} \hat{\mathbf{S}}_{i} \\
& +U \sum_{m i} \hat{n}_{i m \uparrow} \hat{n}_{i m \downarrow}+\sum_{i \sigma \tilde{\sigma}}\left(U^{\prime}-\delta_{\sigma \tilde{\sigma}} J\right) \hat{n}_{i 1 \sigma} \hat{n}_{i 2 \tilde{\sigma}} \\
& +g \sum_{i \sigma} \sum_{m_{1}, m_{2}=1}^{2} \hat{c}_{i m_{1} \sigma}^{\dagger}\left(\hat{Q}_{2} \tau^{z}+\hat{Q}_{3} \tau^{x}\right)_{m_{1} m_{2}} \hat{c}_{i m_{2} \sigma} \\
& +\sum_{a=2}^{3}\left(\frac{1}{2} \hat{P}_{a}^{2}+\frac{1}{2} \Omega^{2} \hat{Q}_{a}^{2}\right)
\end{aligned}
$$

Here, $\hat{c}_{i m \sigma}^{\dagger}$ and $\hat{c}_{i m \sigma}$ are creation and annihilation operators for electrons on site $i$ within $e_{g}$ orbital $m$ and spin $\sigma ; \hat{\mathbf{s}}_{i \nu}=\frac{1}{2} \sum_{\sigma \sigma^{\prime}} \hat{c}_{i \nu \sigma^{\dagger}}^{\dagger} \tau_{\sigma \sigma^{\prime}} \hat{c}_{i \nu \sigma^{\prime}}$ denotes the $\mathrm{e}_{g^{-}}$-spin 
( $\tau$ : Pauli matrices), $\hat{\mathbf{S}}_{i}$ the $\mathrm{t}_{2 g}$-spin, and $\hat{Q}_{a}\left(\hat{P}_{a}\right)$ the coordinate (momentum) of the two quantum Jahn-Teller phonons. Let us briefly discuss the three lines of Hamiltonian (1). The first line describes the Kondo lattice model consisting of two terms: (i) the tight binding band structure with directionally dependent nearest-neighbor hopping, $t_{11}^{x}=-\sqrt{3} t_{12}^{x}=-\sqrt{3} t_{21}^{x}=3 t_{22}^{x}=3 t_{0} / 4, t_{11}^{y}=\sqrt{3} t_{12}^{y}=$ $\sqrt{3} t_{21}^{y}=3 t_{22}^{y}=3 t_{0} / 4, t_{22}^{z}=t_{0}$, and $t_{11}^{z}=t_{12}^{z}=t_{21}^{z}=0$ with bandwidth $W=6 t_{0}=3.6 \mathrm{eV}$ which well describes the LDA band structure for the cubic lattice (contributions of longer-range hopping are very minor [22]); and (ii) the coupling $\mathcal{J}$ to the $t_{2 g}$ spin which we assume to be classical with strength $2 \mathcal{J}\left|\hat{\mathbf{S}}_{i}\right|=2.66$ calculated from a ferromagnetic LDA calculation [17]. The Kondo lattice model (first line) can be solved exactly in DMFT 23. and gives rise to the double exchange mechanism [3] for ferromagnetism. The second line describes the Coulomb interactions between the $e_{g}$ electrons including the intra$(U)$ and inter-orbital repulsion $U^{\prime}$, as well as the Hund's exchange $(J)$. Even for large $\mathcal{J}$, the Coulomb repulsion $U^{\prime}-J$ between two spin-aligned $e_{g}$ electrons on the same lattice site is important. It leads to the formation of Hubbard bands and quasiparticle peaks in the PM phase 14. We take $U^{\prime}=3.5 \mathrm{eV}$ and $J=0.75 \mathrm{eV}$ from Ref. 11] $\left(U=U^{\prime}+2 J\right.$ follows by symmetry). Finally, the third line describes the coupling $g$ of the $e_{g}$ electrons to the two local Jahn-Teller phonons; and the fourth line the quantum motion of these phonons. The phonon frequency $\Omega=0.07 \mathrm{eV}$ is estimated from Raman spectra and the results of lattice dynamical calculations 24]; the breathing mode, which has a higher frequency and is believed to be less important [8], is neglected. Since the electron-phonon coupling is difficult to determine from band structure data, we consider different values, mostly $g=0.10 \mathrm{eV}^{3 / 2}$ which corresponds to a dimensionless coupling constant $\lambda=g / \Omega \sqrt{t}=1.84$, comparable-slightly larger - than previous estimates, see [8]. In the following, we set $\hbar=e=k_{B}=1$; our unit of energy is $\mathrm{eV}$.

$D M F T(Q M C)$ implementation. For solving Hamiltonian (1) by DMFT(QMC), the imaginary time $\tau=0 . . \beta(\equiv$ $1 / T)$ is discretized into $l=1 . . L$ Trotter slices $\tau_{l}$ of width $\Delta \tau=0.2$, and the Coulomb interactions are decoupled through discrete Hubbard-Stratonovich transformations. We sample the auxiliary Hubbard-Stratonovich field and the continuous phonon Bose field $Q_{a}\left(\tau_{l}\right)$ on equal footing, after replacing the phonon momentum $P_{a}\left(\tau_{l}\right)$ by $\left[Q_{a}\left(\tau_{l}\right)-Q_{a}\left(\tau_{l-1}\right)\right] / \Delta \tau$ as discussed in [25]. After each QMC sweep, a global update of the phonon field is considered; we use $\sim 10^{6}-10^{7}$ sweeps.

Polaron formation in the PM phase. Let us start our discussion with the QMC distribution of the $\tau$-averaged phonon field $Q_{2}$, corresponding to the amplitude of the lattice distortion [26]. Fig. 1] shows three peaks with large (positive and negative) Jahn-Teller distortion and small Jahn-Teller distortion, respectively. Upon lowering temperature, and at strong enough electron-phonon

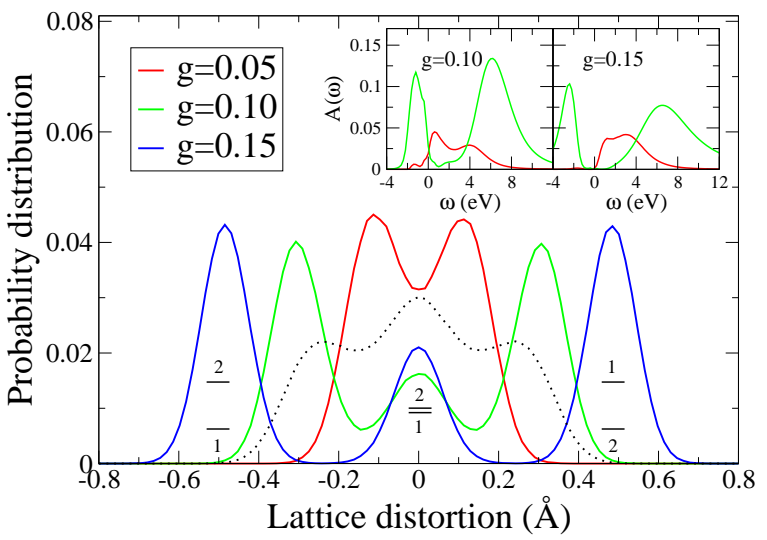

FIG. 1: (Color online) QMC distribution of the lattice distortion $\left[1 / L \sum_{l=1}^{L} Q_{2}\left(\tau_{l}\right)\right]$ for different electron-phonon couplings $g, n=0.8$ electrons/site and $\beta=16$; the dotted line is without Coulomb repulsion for comparion $(g=0.1)$. At strong enough $g$, three regions with large (positive and negative) and small lattice distortion emerge. The inset shows the electron spectral function for these regions with large (light/green) and small (dark/red) lattice distortions, respectively. We can interpret the results physically as the trapping of the $n=0.8$ electrons as Jahn-Teller polarons.

coupling, these three peaks get sharper and clearly separated in the PM phase. Then, the QMC sampling tends to stay within one of the three regions for a very long computational time [27].

Physically, the three sharp peaks mean that the local lattice site is either strongly Jahn-Teller distorted (with orbital 1 or 2 lower in energy as visualized in Fig. 1) or not (central peak). This clear separation into three peaks furthermore suggests that the phonon dynamics is strongly reduced. In our view, the slow QMC dynamics for going from one of the two distorted to the undistorted configurations indeed reflects a slow real time dynamics 28]. Turning to the electronic spectrum in the inset of Fig. 1] we see there is one electron with Jahn-Teller distortion (the peak below $E_{F}=0$ of the light/green line contains one electron) and almost none without distortion (the dark/red line is above the Fermi energy). This is exactly the picture of a local polaron, an electron trapped through the lattice distortion. This trapping explains why we obtain an insulating-like spectrum at $g=0.1$ and even a true gap for $g=0.15$. Let us note that the polaron formation is strongly supported by the local Coulomb repulsion. Without $U^{\prime}$, the polaron formation fades away (dotted line in Fig. 1). The effect of $U^{\prime}$ on the polaron formation is two-fold: (i) It strongly enhances the JahnTeller splitting from $2 E_{\mathrm{JT}}=g^{2} / \Omega^{2}$ to $2 E_{\mathrm{JT}}+U^{\prime}-J$, see the (light/green) spectrum in the presence of distortion in the inset of Fig. 11 Since this splitting has to overcome the $e_{g}$ bandwidth for a polaron localization, $U^{\prime}$ is actually needed - without $U^{\prime}$ no strong lattice distortion emerges (dotted line). (ii) Due to strong quasiparticle renormalizations the effective bandwidth of the correlated bands is 


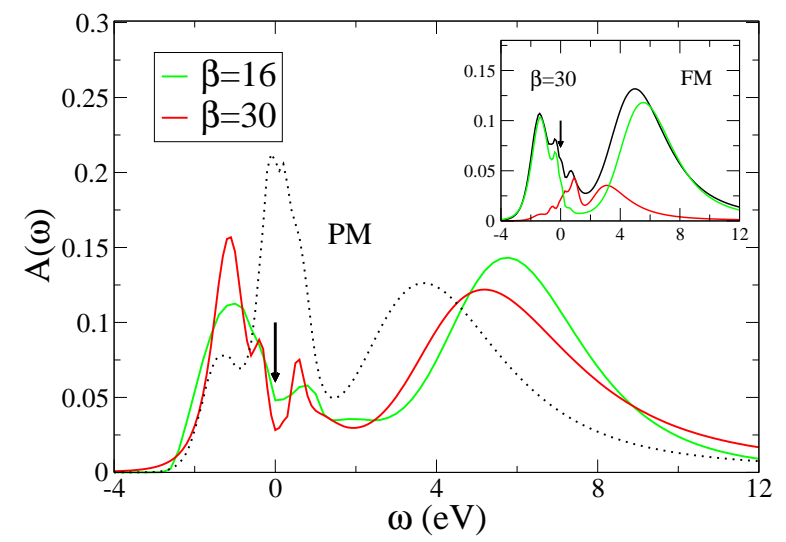

FIG. 2: (Color online) PM spectrum as a function of temperature for $g=0.1$ and $n=0.8$. With decreasing $T$, the polaron formation is stabilized and a pseudo-gap opens at $E_{F}=0$. The dotted line is the PM spectrum without electron phonon coupling at $n=0.8, \beta=30$, showing a damped and renormalized quasiparticle peak at $E_{F}$. Inset: total FM spectrum in the presence (light/green) and absence (dark/red) of lattice distortion, analogous to the inset of Fig. 1 for the PM phase. Due to the enhanced mobility in the FM phase, the Jahn-Teller distorted band (i.e., the lower light/green band) is wider, not fully occupied, and hence metallic-like.

strongly reduced (see the dotted line in Fig. 2). We can estimate this bandwidth renormalization to be roughly $Z=\left(1-\partial \operatorname{Re} \Sigma(\omega) /\left.\partial \omega\right|_{\omega=0}\right)^{-1} \sim 0.4$, as obtained from the self energy at real $\omega$. Note, however, that these quasiparticles are strongly damped through scattering at $t_{2 g}$ spins $(\operatorname{Im} \Sigma(0) \sim-0.7 \mathrm{eV})$. Since the correlated electrons are already more localized, it is easy to trap them as polarons.

Spectrum in the PM phase. Let us turn to the total (distortion-averaged) PM spectrum in Fig. 22 It shows, upon decreasing $T$, the development of a pseudogap at $E_{F}$ which separates localized Jahn-Teller polarons and undistorted configurations without electrons. This pseudogap is more enhanced at lower $T$ since - without thermal smearing of the lattice distortion - the three peaks in Fig. 1 become sharper. Consequently, there is a sharper separation (pseudogap) between polaron trapped electrons and undistorted unoccupied states.

In Fig. 3. we see that the pseudogap of the PM spectrum leads to a similar pseudogap in the optical spectrum at small frequencies, so that the optical spectral weight is very much reduced below $1 \mathrm{eV}$ [26]. The first optical peak at $\sim 1.5 \mathrm{eV}$ (named midgap state in the literature) then stems from exciting the polarons into the unoccupied, undistorted band above the Fermi energy. At $\sim 5-6 \mathrm{eV}$, a second peak in the optical spectrum signals the excitations of states with two $e_{g}$ electrons per site, which correspond to the upper (light/green) peak in the inset of Fig. 11 The reduced optical spectral weight at low frequencies, and (approximately) the position of the two optical peaks are in agreement with experiment 12, as is

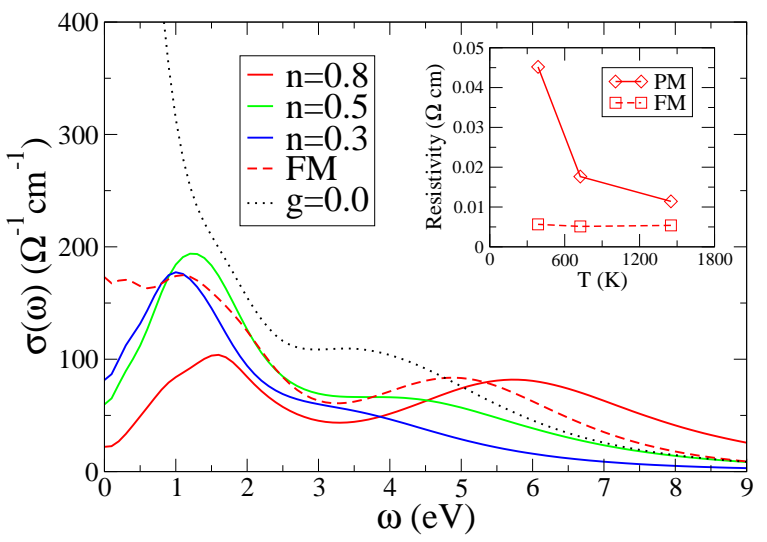

FIG. 3: (Color online) Optical conductivity $\sigma(\omega)$ for the PM phase at $g=0.1, \beta=30$ and $n=0.8,0.5$, and 0.3 electrons/site, showing an insulating-like behavior with a reduced weight below $1 \mathrm{eV}$. The dotted line shows a metallic Drude peak in the absence of the electron-phonon coupling $(\beta=30, n=0.8)$; the dashed line the bad metallic behavior of the FM phase $(g=0.1, \beta=30, n=0.8)$. Inset: The PM resistivity strongly increases with decreasing $T$ (here for $g=0.1$ and $n=0.8$ ) so that a transition to the FM phase reduces the resistivity by a factor of $\sim 8$. There is a "colossal" magnetoresistance.

the shift of the two peaks towards lower frequencies with increasing hole doping $x$ (decreasing $n=1-x$ ) and the gradual filling of the optical gap with increasing $x$ 12]. Let us note that the height of the two optical peaks does not fully agree with experiment: In contrast to Fig. 3 the second peak has experimentally several times more spectral weight than the first (midgap) peak. We can understand this disagreement since there are other optical contributions in this energy range: from oxygen charge transfer, from La bands, and the upper Hubbard band of the $t_{2 g}$ electrons. Such effects are beyond our low-energy Hamiltonian (11). Also the first (midgap) peak has experimentally a larger $\sigma(\omega)$ than in Fig. B for a smaller $g, \sigma(\omega)$ would increase and the two peaks would shift to lower $\omega$.

From $\sigma(0)$, we directly obtain the resistivity in the inset of Fig. 3 As the electrons are more strongly trapped as Jahn-Teller polarons with decreasing temperature, the resistivity of the PM phase is strongly enhanced. We observe an insulating-like behavior.

(Bad) metallic FM phase. Let us finally turn to the FM phase which we simulate by fixing the direction of the $t_{2 g}$ spins. The FM spectrum for $n=0.8$ and $\beta=30$ is presented in the inset of Fig. 22 separated into contributions from small and large Jahn-Teller distortion. While the peak positions of the distorted and undistorted bands are at similar energies as for the PM in Fig. [1] the widths of these bands are much larger so that the band with Jahn-Teller distortion crosses the Fermi level and is hence metallic-like. This is also reflected in the self-energy which, in contrast to the PM phase, behaves metallic-like with $\partial \operatorname{Re} \Sigma(\omega) / \partial \omega<0$, corresponding to a 
quasiparticle weight of roughly $Z \sim 0.6$. However, these quasiparticle excitations are very strongly damped since, when moving through the crystal, they scatter at different local potentials, with and without Jahn-Teller distortion. The scattering rate is given by $\operatorname{Im} \Sigma(0) \sim-1.9 \mathrm{eV}$ which corresponds to a quasiparticle life time of half a femto second. It is hence questionable whether we can still speak of quasiparticles at all. In any case, a bad metallic behavior is to be expected and indeed seen in the optical conductivity [12], showing a wide peak at low frequencies (dashed line in Fig. 3).

Having described the insulating-like PM with a pseudogap in the spectrum and the FM without this pseudogap, the magnetoresistance simply follows from the PM-FM transition-triggered by temperature or magnetic fields. For $n=0.8$, the inset of Fig. 3] yields a change of resistivity by about a factor 8 when going from the PM to the FM phase.

Conclusion. We reported how the tendencies of Coulomb repulsion and Jahn-Teller distortion to localize electrons mutually enhance each other so that correlated electrons are trapped as polarons in the PM phase of doped manganites. In the FM phase, on the other hand, electrons are still mobile, but strongly scattered because of fluctuating (strong and weak) Jahn-Teller distortions, yielding a bad metal. The transition from PM to FM is hence accompanied by a "colossal" magnetoresistance.

We thank O. K. Andersen, N. Blümer, N. N. Kovaleva, O. Rösch, A. Yamasaki, and particularly J. E. Han, who explained to us how he treats phonons in QMC, for discussions; and the Deutsche Forschungsgemeinschaft for financial support through the Emmy Noether program.

[1] R. von Helmolt et al., Phys. Rev. Lett. 71, 2331 (1993); S. Jin et al., Science 264, 413 (1994); P. Schiffer et al., Phys. Rev. Lett. 75, 3336 (1995).

[2] A. P. Ramirez, J. Phys.: Condens. Matter 9, 8171 (1997); Y. Tokura and Y. Tomioka, J. Magn. Magn. Mater. 200, 1 (1999); M. B. Salamon and M. Jaime, Rev. Mod. Phys. 73, 583 (2001).

[3] C. Zener, Phys. Rev. 82, 403 (1951); P. W. Anderson and H. Hasegawa, Phys. Rev. 100, 675 (1955).

[4] A. J. Millis, P. B. Littlewood, and B. I. Shraiman, Phys. Rev. Lett. 74, 5144 (1995); H. Röder, J. Zang, and A. R. Bishop, Phys. Rev. Lett. 76, 1356, (1996); A. J. Millis, B. I. Shraiman, and R. Mueller, Phys. Rev. Lett. 77, 175 (1996).

[5] R. Kilian and G. Khaliullin, Phys. Rev. B 58, 11841 (R) (1998); P. Horsch, J. Jaklič, and F. Mack, Phys. Rev. B 59, 6217 (1999).

[6] C. M. Varma, Phys. Rev. B 54, 7328, (1996).

[7] S. Yunoki, A. Moreo, and E. Dagotto, Phys. Rev. Lett. 81, 5612 (1998).

[8] E. Dagotto, T. Hotta, and A. Moreo, Physics Reports 3441 (2001).

[9] T. V. Ramakrishnan et al., Phys. Rev. Lett. 92, 157203
(2004)

[10] A. E. Bocquet et al., Phys. Rev. B 46, 3771 (1992); A. Chainani, M. Mathew, and D. D. Sarma, Phys. Rev. B 47, 15397 (1993); T. Saitoh et al., Phys. Rev. B 56, 8836 (1997).

[11] J.-H. Park et al., Phys. Rev. Lett. 76, 4215 (1996).

[12] Y. Okimoto et al., Phys. Rev. Lett. 75, 109 (1995); M. Quijada et al., Phys. Rev. B 58, 16093 (1998); J. H. Jung et al., Phys. Rev. B 57, R11 043 (1998); K. Takenaka et al., J. Phys. Soc. Jpn. 68, 1828 (1999); N. N. Kovaleva et al., Phys. Rev. Lett. 93, 147204 (2004).

[13] E.g., M. Imada, J. Phys. Soc. Jap. 67, 45 (1998); M. J. Rozenberg, Eur. Phys. J. B 2, 457 (1998); P. Benedetti and R. Zeyher, Phys. Rev. B 59, 9923 (1999); Y. Motome and M. Imada, J. Phys. Soc. Jap. 68, 16 (1999); Th. Pruschke and M. B. Zölfl, Adv. Solid State Phys. 40, 251 (2001); M. Daghofer, A. M. Oleś, and W. von der Linden, Phys. Rev. B 70, 184430 (2004); M. S. Laad, L. Craco, and E. Muller-Hartmann, New J. Phys. 6, 157 (2004).

[14] K. Held and D. Vollhardt, Phys. Rev. Lett. 84, 5168 (2000).

[15] V. I. Anisimov et al., J. Phys.: Condens. Matter 9, 7359 (1997); A. I. Lichtenstein and M. I. Katsnelson, Phys. Rev. B 57, 6884 (1998); for reviews see K. Held et al., Psik Newsletter \#56 (2003); cond-mat/0511293 G. Kotliar et al., cond-mat/0511085

[16] I. Loa et al., Phys. Rev. Lett. 87, 125501 (2001).

[17] A. Yamasaki et al., cond-mat/0602358

[18] W. Metzner and D. Vollhardt, Phys. Rev. Lett. 62, 324 (1989); A. Georges et al., Rev. Mod. Phys. 68, 13 (1996).

[19] J. E. Hirsch and R. M. Fye, Phys. Rev. Lett. 56, 2521 (1986).

[20] A. J. Millis, R. Mueller, and B. I. Shraiman, Phys. Rev. B 54, 5405 (1996).

[21] B. Michaelis and A. J. Millis, Phys. Rev. B 68, 115111 (2003).

[22] A. Yamasaki et al. (unpublished).

[23] N. Furukawa, J. Phys. Soc. Jap. 63, 3214 (1994).

[24] M. N. Iliev et al., Phys. Rev. B. 57, 2872 (1998).

[25] R. Blankenbecler, D. J. Scalapino, and R. L. Sugar, Phys. Rev. D 24, 2278 (1981); J. E. Han, Phys. Rev. B 70, 054513 (2004).

[26] We use the mass of the oxygen atom for getting the lattice distortion in $\AA$ in Fig. 1] and the lattice constant $a=$ $5.5 \AA$ for getting the optical conductivity in Fig. 3

[27] In the extreme cases $(g=0.15 ; \beta=16)$ and $(g=0.1$; $\beta=30)$, it is easy to QMC sample the regions of the three different peaks independently. But because of the extremely low propability to go from one region to another, the relative importance of the three well-separated regions is not adequately sampled by QMC. This relative weight can, on the other hand, be extrapolated from high $T$, which agrees with the physical expectation that the weight is $1-n$ and $n / 2$ for small distortion and the two regions with large distortions, respectively. For the final results for the above-mentioned cases, we hence weight the independently sampled regions accordingly.

[28] Note that a very similar (two maxima) distribution of the averaged Ising-like field is observed for the DMFT Mott insulator, where it indicates the presence of a localized spin (up or down); and the slow QMC dynamics from spin up to down reflects the long-time stability of the spins in the Mott-insulating phase of the Hubbard model. 